\title{
An Inductive Link-Based Wireless Power Transfer System for Biomedical Applications
}

\author{
M. A. Adeeb, ${ }^{1,2}$ A. B. Islam, ${ }^{1}$ M. R. Haider, ${ }^{3}$ F. S. Tulip, ${ }^{4}$ M. N. Ericson, ${ }^{4}$ and S. K. Islam ${ }^{1}$ \\ ${ }^{1}$ Department of Electrical Engineering and Computer Science, The University of Tennessee, Knoxville, TN 37996, USA \\ ${ }^{2}$ RF MicroDevices, Inc., Greensboro, NC 27409, USA \\ ${ }^{3}$ Department of Engineering and Computer Engineering, The University of Alabama, Birmingham, AL 35294, USA \\ ${ }^{4}$ Oak Ridge National Laboratory, Oak Ridge, TN 37831-6006, USA \\ Correspondence should be addressed to A. B. Islam, aislam@utk.edu
}

Received 14 November 2011; Revised 19 February 2012; Accepted 5 March 2012

Academic Editor: Sheng Lyang Jang

Copyright (๑) 2012 M. A. Adeeb et al. This is an open access article distributed under the Creative Commons Attribution License, which permits unrestricted use, distribution, and reproduction in any medium, provided the original work is properly cited.

A wireless power transfer system using an inductive link has been demonstrated for implantable sensor applications. The system is composed of two primary blocks: an inductive power transfer unit and a backward data communication unit. The inductive link performs two functions: coupling the required power from a wireless power supply system enabling battery-less, long-term implant operation and providing a backward data transmission path. The backward data communication unit transmits the data to an outside reader using FSK modulation scheme via the inductive link. To demonstrate the operation of the inductive link, a board-level design has been implemented with high link efficiency. Test results from a fabricated sensor system, composed of a hybrid implementation of custom-integrated circuits and board-level discrete components, are presented demonstrating power transmission of $125 \mathrm{~mW}$ with a $12.5 \%$ power link transmission efficiency. Simultaneous backward data communication involving a digital pulse rate of up to $10 \mathrm{kbps}$ was also observed.

\section{Introduction}

Recent progress in biomedical sensing technologies and proliferation of micro- and nano-fabrication facilities with inexpensive signal processing systems has led to the development of various biomedical sensors. Silicon-based microfabrication and microelectromechanical techniques have been successfully adopted for fabrication of a large range of miniature electrochemical biomedical sensors. These advances in the fabrication process technologies have enabled significant recent research focused on the investigation of continuous in vivo measurement and monitoring of various physiological variables by means of an implantable sensor $[1,2]$. Examples include monitoring of blood glucose level [3-5], continuous in vitro monitoring of lactate in bloodstream or tissues [6], and minimally invasive pressure monitoring of blood vessels and intracranial compartments [7]. A summary of the primary emerging and commercialized sensing technologies of the past few years has been presented by Coté et al. [8].
For biomedical applications, implanted electronics are being increasingly used for real-time patient monitoring, diagnosis, and in some cases, treatment of certain medical conditions. In case of implantable sensors, powering up the devices is one of the most important concerns. Inductive link is a common method for wireless powering of implanted biomedical electronics and data communication with the external world. Previously, transcutaneous power cables were used in some clinical implantable applications [9], but they introduce a significant path for infection. One biomedical to the transcutaneous power cables is the use of implanted batteries which provide a limited supply of power and may exceed size and mass requirements for the implant. In addition, replacement can only be performed by surgical procedure, and long-term implantation introduces a potential risk of leakage. Alternatively, inductive links do not suffer from these limitations and consequently produce increased implant robustness, and if implemented properly, provide sufficient miniaturization. 
Wireless power transmission and data communication using an inductive link have been demonstrated for various biomedical applications including visual prosthesis, cochlear implants, neuromuscular and nerve stimulators, cardiac pacemakers/defibrillators, deep-brain stimulators, spinalcord stimulators, brain-machine interfaces, gastrointestinal microsystems, and capsule endoscopy [10-19]. Much of the published research works utilize circuits implemented using low-cost commercial off-the-shelf (COTS) components allowing more rapid prototyping and validation of inductive link systems. Turgis and Puers reported a board-level design of radio telemetry concept using COTS components for capsule endoscopy [20]. Catrysse et al. presented an inductive link using COTS components with bidirectional data transmission system for biomedical application delivering $50 \mathrm{~mW}$ over a $3 \mathrm{~cm}$ distance at $700 \mathrm{kHz}$ [21]. Ghovanloo and Najafi presented a system-on-chip (SOC) concept by combining ASIC design with off-chip components (LC tank; filters) for biomedical application delivering $50 \mathrm{~mW}$ over a $5 \mathrm{~mm}$ distance at 5/10 MHz frequency [22]. Li and Bashirullah also demonstrated an ASIC-based approach with the inductive coils as off-chip components delivering $6.15 \mathrm{~mW}$ at $4 \mathrm{MHz}$ [23].

Two other important parameters related to wireless power transmission in biomedical applications are the frequency of operation and the data rate. Federal Communications Commission (FCC) regulates the time and the amount of exposure of electromagnetic waves to human tissues at various frequencies [24]. In addition to human tissue compatibility, wireless-based systems require noninterference with existing communication systems. Due to these stringent requirements, medical radios tend to use industrial, scientific, and medical (ISM) frequency bands with low data rate operation. Depending on the application, medical radios use various data rates and frequencies. For example, data bandwidths for pacemakers, cardiac defibrillators, and analog cochlear processors are typically around $8 \mathrm{kbps}$ $[25,26]$, neural recording uses $800 \mathrm{kbps}$ [27], and retinal stimulators use $40 \mathrm{kbps}$ [28].

In this paper, we present an inductive powering and backward data communications link based on COTS components. The inductive power link with backwards data communication is demonstrated using a board level implementation of the power link and a data modulator unit composed of COTS components. The inductive link is capable of delivering $125 \mathrm{~mW}$ at $5 \mathrm{~V}$ with appreciable overall power transmission efficiency. This is accomplished by using an efficient class $\mathrm{E}$ type switching power amplifier or driver. The operating frequency was chosen to optimize power transfer while minimizing tissue electromagnetic field exposure. Test results show that the prototype inductive link can transmit power to a distance of $1.16 \mathrm{~cm}$ with overall link efficiency of $12.5 \%$. Future efforts will be directed towards further optimization and miniaturization of the system.

\section{Inductive Link Design}

The inductive link was designed using COTS components for validation of the concept of inductive powering with backwards data transmission. The inductive link consists of two coaxially aligned circular coils operating as a weakly coupled air core transformer, of which one coil resides inside the human body, while the other is placed in an external unit on top of the skin. The powering system mainly consists of two units: an external primary power system unit (power transmitter) and an internal secondary power system unit (power receiver). The external unit functions as the power source of the internal unit and also serves as a data receiver from the internal unit enabling backward data communication. The internal unit receives processed sensor data from the sensor signal conditioning circuit, and after converting to an FSK modulated signal, sends the sensor data back to the external unit. An overview of these two units and the design considerations of a class E power amplifier for driving the primary coil are presented in the following sections (see Figure 1).

\subsection{Design Considerations}

2.1.1. The Coil System. The circular coil system acts as a weakly coupled air-core transformer between the external (primary) and the internal (secondary) parts of the system and consists of two hand-wound coils of suitable geometri$\mathrm{cal}$, magnetic, and electric properties. The selection of different parameters is performed based on physical constraints, performance and compatibility with other system elements.

2.1.2. Choice for Secondary Resonance. It has been reported in [21] that either series or parallel resonance can be employed to achieve optimal coupling efficiency. For a given set of coil quality factors $(Q)$ and low-coupling factor (hence lowefficiency links), it has been shown [29] that the series resonance topology requires a very large secondary coil inductance to achieve optimum link efficiency. These large inductance values may be practically impossible in terms of implementation, as there are size limitations for the internal secondary coil in most applications including implantable biomedical sensor applications. Therefore, low-power links are usually utilized using secondary parallel resonance, making the LC tank act as a voltage source. For highpower, high-coupling applications, it has been shown by Vandevoorde and Puers [29] in a similar fashion that both resonance schemes are practically realizable and can be quite efficient as well.

2.2. Primary-Side Class E Driver Design. A class E power amplifier was employed in the primary unit to drive the link coils (see Figure 2) which is a widely used topology for biomedical applications. This is because theoretically class $\mathrm{E}$ amplifiers can reach power efficiencies approaching 100\% $[30,31]$. The frequency of operation was selected to be $200 \mathrm{kHz}$ which conforms to the current density limit set by NCRP (National Council on Radiation Protection and Measurements) regulation of maximum exposure of human tissue to radio frequency electromagnetic field [32]. One of the leading design factors in a class E power amplifier or driver design is the quality factor of the load network $\left(Q_{L}\right)$. 


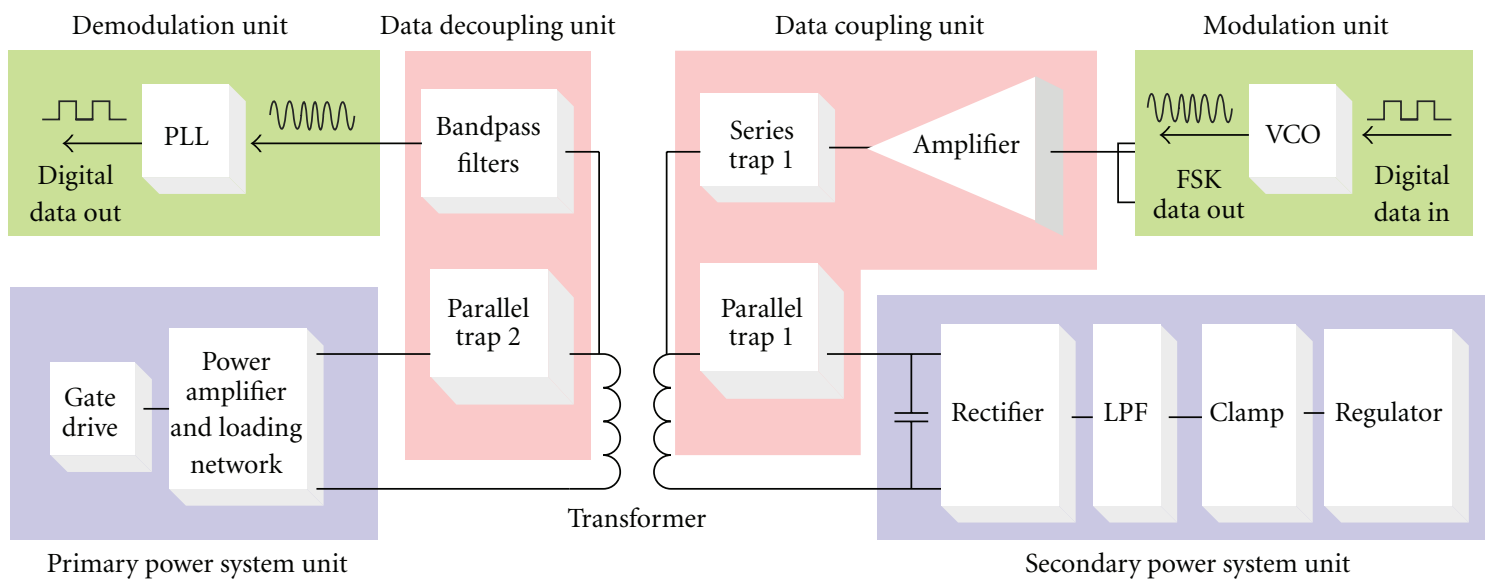

FIgURE 1: Detailed system block diagram of inductive link.

The assumption of a sinusoidal current in $L_{1}$ and $C_{1}$ is accurate only if $Q_{L}$ is infinite. In addition, the output power increases as $Q_{L}$ increases. Therefore, the choice of $Q_{L}$ involves a trade-off between the operating bandwidth (wider with lower $Q_{L}$ ), the harmonic content of the output power (lower with higher $Q_{L}$ ) and the power loss in the parasitic resistance of the load-network inductor $L_{1}$ and capacitor $C_{1}$ (lower with lower $Q_{L}$ ) [33]. The purpose of the proposed inductive link is to provide not only the power to the implantable electronics, but also a means for data communication from the internal unit to the external unit, and hence a high $Q_{L}$ is not suitable. Therefore, $Q_{L}$ was selected to be less than 5 .

Reference [34] summarizes design equations and associated methodology for choosing the loading network component values of a class $\mathrm{E}$ power amplifier for a given load resistance $\left(R_{L}\right)$, quality factor of the loading network $\left(Q_{L}\right)$, and operating frequency $(f)$. The total load resistance seen by the amplifier is calculated as

$$
R=R_{L}+\mathrm{ESR}_{\mathrm{L} 1}+\mathrm{ESR}_{C 1}+1.365 R_{\mathrm{on}}+0.2116 \mathrm{ESR}_{C S}
$$

where $R_{\text {on }}$ is the on-resistance of the power transistor, and ESR is the effective series resistance of a reactive component.

The design equations are as follows [31]:

$$
\begin{aligned}
C_{S}= & \frac{1}{2 \pi f R\left(\pi^{2} / 4+1\right) \pi / 2}\left(0.99866+\frac{0.91424}{Q_{L}}-\frac{1.03175}{Q_{L}^{2}}\right) \\
& +\frac{0.6}{(2 \pi f)^{2} L_{D}}, \\
C_{1}= & \frac{1}{2 \pi f R}\left(\frac{1}{Q_{L}-0.104823}\right)\left(1.00121+\frac{1.01468}{Q_{L}-1.7879}\right) \\
& -\frac{0.2}{(2 \pi f)^{2} L_{D}},
\end{aligned}
$$

$$
L_{1}=\frac{Q_{L} R}{2 \pi f} .
$$

Usually, $L_{D}$ is chosen such that $X_{L D}$ is 30 or more times the unadjusted value of $X_{C S}$.

In the work presented in this paper, the class $\mathrm{E}$ amplifier does not drive a simple resistive load; rather it drives a transformer loaded with some power management blocks, which are mostly nonlinear in nature. However, in order to make use of the design equations mentioned here, an estimation of the load resistance $\left(R_{L}\right)$ that is seen by the primary loading network is required. Applying transformer principles, the reflected secondary load to the primary side can be found using the following equations:

$$
\begin{gathered}
R_{L}=\frac{(\omega M)^{2}}{R_{S}}, \\
M=k \sqrt{L_{1} L_{2}}=\frac{V_{S}}{\omega I_{1}},
\end{gathered}
$$

where $I_{1}=$ primary coil current $(\mathrm{rms})$, and $R_{S}$ is the equivalent secondary impedance, which is calculated to be $408.4 \Omega$.

From (5) and (6), it is evident that the effective secondary resistance reflected back to the primary side depends on the mutual inductance between the coils and hence on the self-inductance of the primary coil. Again, the choice of primary coil inductance is dependent upon the choice of $Q_{L}$ and the total resistance seen by the driver $(R)$, of which $R_{L}$ is a part. There are also other considerations that affect the choice of primary coil inductance. Consequently, the design of the loading network for the class E amplifier requires careful consideration of the following issues:

(i) $Q_{L}$ of the loading network should not be greater than 5 , which limits the $L_{1} / R$ ratio;

(ii) $R_{L}$ should make up the major portion of $R$ so that the drain efficiency of the amplifier, and hence the power transfer efficiency is high. Careful selection of a low on-resistance power device, and low ESR reactive elements is needed; 


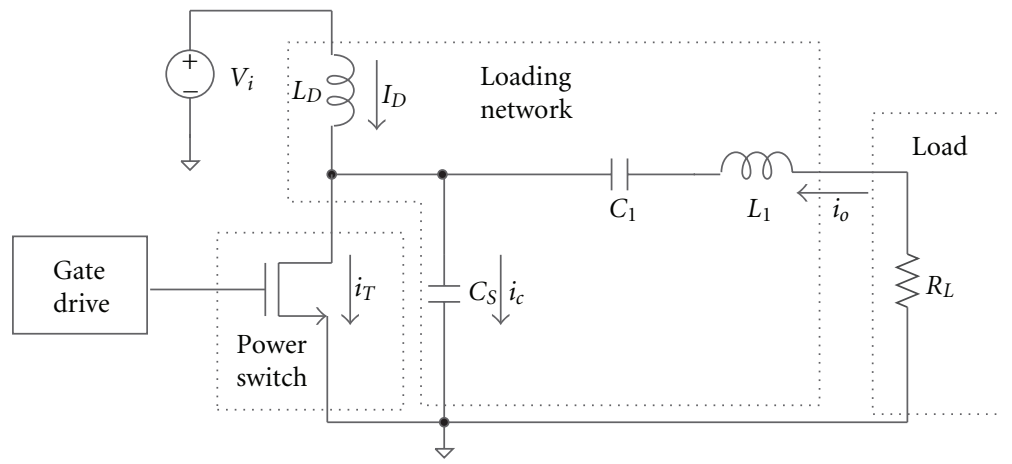

Figure 2: Schematic of a typical class E power amplifier.

(iii) the primary drive circuit current can be large (several hundred milliamps) requiring proper selection of the wire diameter;

(iv) the product of the mutual inductance between the coils, the primary current, and the operating frequency should be sufficiently large to provide the required induced voltage at the secondary tank.

In order to sufficiently address these guidelines, the selection of the coil inductance and the loading network parameters is often an iterative process.

One of the topmost priorities in the link design process was achieving a high-coupling factor for the inductive link. Key to accomplishing this objective was to incorporate a large number of turns in the secondary coil, within the physical limits of the prototype. This was comparatively easy because the current in the secondary unit is much smaller, allowing the use of a thinner (AWG number 30) wire. The ESR of the secondary coil was also relaxed compared to the primary. This allowed having ample self-inductance in the secondary coil $(267 \mu \mathrm{H})$ as well as the provision of obtaining a high mutual inductance without being required to put too many turns in the primary coil.

The primary coil was constructed using AWG number 18 producing an inductance of $5.8 \mu \mathrm{H}$. To better match the calculated inductance, $L_{1}$, a conformal coated inductor of $1.6 \mu \mathrm{H}$ was added, producing a total inductance of $7.4 \mu \mathrm{H}$ with an ESR of $0.29 \Omega$ at $200 \mathrm{kHz}$. Using this value along with $L_{2}$ and $k$ results in $M=0.453 \sqrt{7.4 \times 10^{-6} \times 267 \times 10^{-6}}=$ $20.14 \mu \mathrm{H}$ and $R_{L}=1.57 \Omega$.

High-current, high- $Q$ polypropylene capacitors were chosen for the primary circuit having low ESR $(\sim 0.024 \Omega)$. Using a low on-resistance power MOSFET with $R_{\text {on }}=0.1 \Omega$, (1) produces

$$
\begin{aligned}
R & =1.57+0.29+0.025+1.365 \times 0.1+0.2116 \times 0.025 \\
& =2.03 \Omega .
\end{aligned}
$$

Using the adjusted values of $R$ and $L_{1}, Q_{L}$ was calculated again by (4):

$$
Q_{L}=\frac{2 \pi f L_{1}}{R}=4.58,
$$

which is an acceptable value for the loaded $Q$.

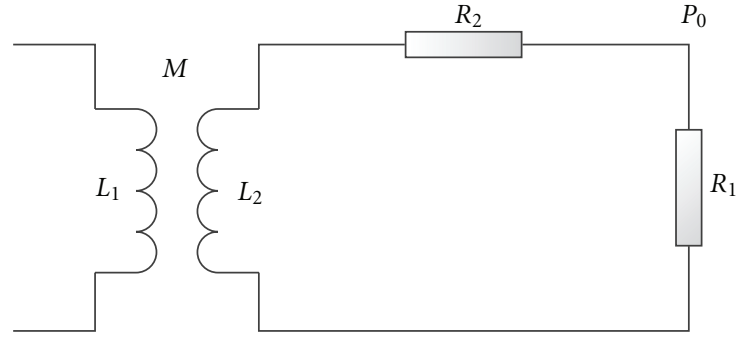

FIgURE 3: Schematic of inductively coupled circuit.

A high-current, high-inductance toroidal RF choke has been chosen as $L_{D}$. For this part, $L_{D}=481 \mu \mathrm{H}$. Substituting this value and the calculated results for $R$ and $Q_{L}$ into (2) and (3) yields $C_{S}=83.5 \mathrm{nF}$ and $C_{1}=119.3 \mathrm{nF}$, respectively. Using the aforementioned values produced $X_{C S}=9.53 \Omega$ and $X_{L D}=604.44 \Omega$ at the $200 \mathrm{kHz}$ operating frequency.

2.3. Design of the Coils. Figure 3 shows a schematic of an inductively coupled circuit.

The mutual inductance $(M)$ of two inductively coupled coils is given by (6). At resonance condition the optimum value of $R_{2}$ required for maximum efficiency can be expressed as [35]:

$$
R_{2 \mathrm{opt}}=\frac{R \sqrt{1+k^{2} Q_{1} Q_{2}}}{Q_{2}^{2}} .
$$

The optimum efficiency is calculated as [35]:

$$
\eta_{\mathrm{opt}}=\frac{k^{2} Q_{1} Q_{2}}{\left(1+\sqrt{1+k^{2} Q_{1} Q_{2}}\right)^{2}} .
$$

The primary design objective was to achieve the maximum coupling coefficient $(k)$ possible under given geometric constraints. For maximum coupling efficiency the primary coil diameter $d_{1}$ is calculated as

$$
d_{1}=\sqrt{d_{2}^{2}+4 d^{2}}
$$

where $d_{1}$ is the diameter of the primary coil, $d_{2}$ is the diameter of the secondary coil, and $d$ is the spacing between 


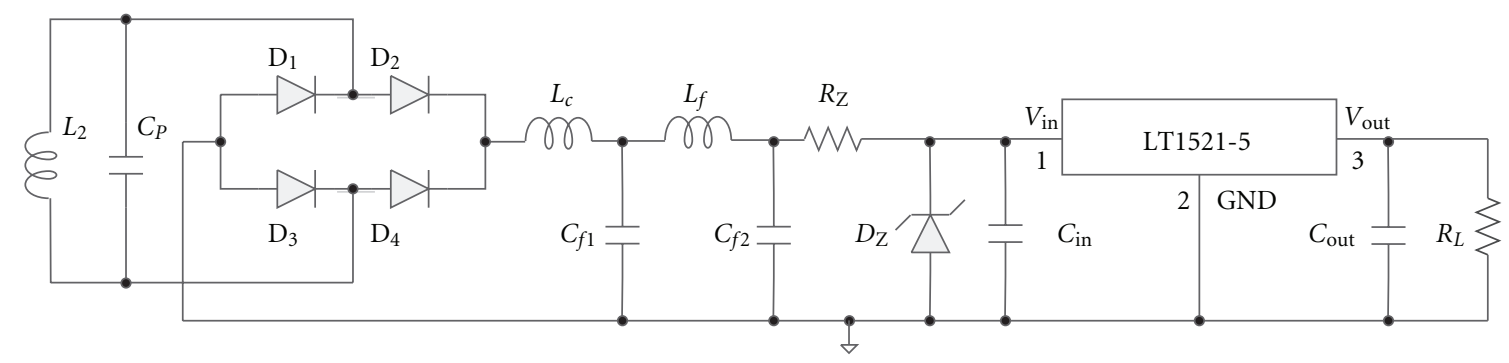

FIgURE 4: Schematic diagram of the inductive powering secondary unit of the system.

TABLE 1: Inductive link specifications.

\begin{tabular}{lc}
\hline Parameters & Value \\
\hline Frequency & $200 \mathrm{kHz}$ \\
Primary coil $\left(L_{1}\right)$ & $7.4 \mu \mathrm{H}$ \\
Secondary coil $\left(L_{2}\right)$ & $267 \mu \mathrm{H}$ \\
Coupling factor $(k)$ & 0.453 \\
Load quality factor $\left(Q_{L}\right)$ & 4.58 \\
Capacitor $\left(C_{S}\right)$ & $83.5 \mathrm{nF}$ \\
Primary capacitor $\left(C_{1}\right)$ & $119.3 \mathrm{nF}$ \\
\hline
\end{tabular}

the coils. The secondary coil diameter, $d_{2}$, is chosen to be $7.12 \mathrm{~cm}$ with the assumption that the coil perimeter will set the size of the implanted system. Using (11), $d_{1}=7.4 \mathrm{~cm}$ is obtained. The secondary coil inductance and the resonant tank capacitance are calculated from the following equations:

$$
\begin{gathered}
L_{2}=\frac{Q_{1} Q_{2}}{2 \pi f}, \\
C_{p}=\frac{1}{(2 \pi f)^{2} L} .
\end{gathered}
$$

Summary of inductive link parameters is given in Table 1.

2.4. Secondary Side Power Conversion and Management. The secondary unit consists of a resonant tank followed by a rectifier, a low-pass filter, a clamp circuit, and a voltage regulator (see Figure 4). A capacitor $\left(C_{p}\right)$ was placed across the secondary coil $\left(L_{2}\right)$ to form a tank circuit that resonates at the operating frequency of the primary drive coil. Due to parallel resonance of the tank, it behaves as an AC voltage source to the rest of the secondary network. A full-wave rectifier and a low-pass filter follow the resonant tank to rectify the induced AC voltage in the secondary tank and then to filter the rectified signal to convert it to a DC signal. A Zener diode $\left(D_{\mathrm{Z}}\right)$-based clamp circuit was used between the low-pass filter output and the regulator input in order to provide overvoltage protection for the regulator input pin. A voltage regulator (LT1521-5) was employed at the final stage of the powering system to provide a regulated supply voltage.

2.5. Backward Data Communication Unit. The sensor is powered inductively from the power transmitter (or data reader), and it transmits the data back to the reader. Lowpower, low-complexity, and low-to-medium data rates are the key requirements for the design of the data transmitter. Amplitude shift keying (ASK) is the most efficient lowpower digital modulation scheme available today, but it suffers from noise susceptibility. To achieve better noise performance, FSK modulation scheme was chosen for the backward data communication system. FSK consumes more power than ASK but achieves excellent noise performance with low design complexity. The modulation unit consists of a VCO where the input to the VCO is the digital data from the sensor signal conditioning circuit. As the VCO output frequency is ideally proportional to the magnitude of its signal input, it generates two different frequencies for the logic "0" ("Space") and logic "1" ("Mark") levels, where the separation is separated by an amount depending on the VCO gain.

The CD4046B CMOS low-power PLL chip from Texas Instruments was used as the FSK modulator for this work. This versatile IC consists of a linear VCO and two different phase comparators having a common signal-input amplifier and a common comparator input and can operate with frequencies up to $1.2 \mathrm{MHz}$. Only the VCO portion of this chip was used with proper timing resistors and capacitors to generate the FSK frequencies.

The 4046 PLL chip is not capable of driving a lowimpedance reactive load, while the data signal should not interfere with the secondary power management blocks. For these two considerations, a gain stage and suitable trapping networks were used in the implantable unit to facilitate the data coupling with the secondary coil. A resonant tank circuit behaves as an inductor below the frequency of resonance and as a capacitor above the frequency of resonance. It has been found that the inductive reactance of the parallel secondary tank is less than $500 \Omega$ at the FSK center frequency. Therefore, a simple common-source gain stage has been added at the output of the modulator (VCO) (see Figure 5). The gain setting resistor was chosen to be $1 \mathrm{k} \Omega$, so that the current to the amplifier does not exceed $5 \mathrm{~mA}$ with a $5 \mathrm{~V}$ internal supply. An $n$-channel vertical MOSFET (ZVN0120A) has been used as the active device. The output of the commonsource amplifier has been connected to the secondary coil through a series resonant network, known as a series trap, so that it offers a low-impedance path for the FSK signals at their center frequency. A parallel trap comprising of $L_{t 1}$ and $C_{t 1}$ has been used between the secondary coil and the parallel 


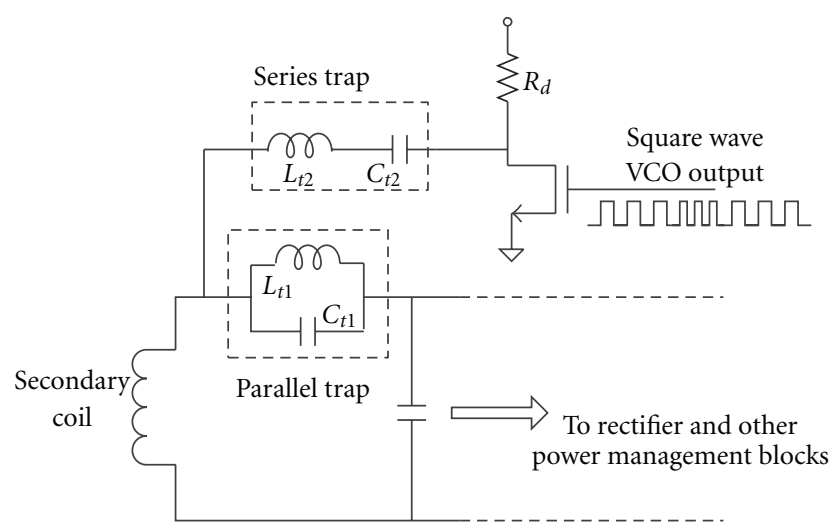

FIGURE 5: Gain stage and the trap networks.

resonating capacitor by breaking up the loop. This trap isolates the data signal from the rest of the power recovery circuitry by providing high impedance at the mark and space frequencies, and thus keeps the efficacy of the power recovery unit virtually unaffected.

However slight deviations in operating frequency due to the poor tolerance of the reactive component values and additional parasitics may shift the resonant condition and result in poor performance of the trap networks. As a result, the trap networks were implemented using high-precision components to minimize errors.

The CD4046B PLL chip has also been used to implement the FSK demodulator. The demodulator design involves the proper choice of timing resistors-capacitors and the loopfilter elements. As can be seen from the schematic diagram of the demodulator as illustrated in Figure 6, the filtered FSK signal is ac-coupled to the signal input of the phase comparator. The VCO frequencies are set the same as the ones of the modulator, and the VCO output is connected to the phase comparator. Between the output of the phase comparator (PC) and the VCO input, a passive R-C low-pass filter is used to reject the ripple at the PC output.

\section{Measurements and Test Results}

The external and the internal units of the inductive powering and data modulator system were implemented on printed circuit boards (PCBs) using discrete components. Keeping the main supply voltage constant, the design parameters were varied. Both the primary and the secondary coils were built by winding several turns of AWG number 18 and number 30 wires with coupling coefficient $(k)$ very close to the value of 0.453. The electrical parameters of the coils were measured using an HP 4192A LF Impedance Analyzer, and these parameters were used for calculating the system performance. Measurements are carried out to observe the effect of the change in frequency, coil spacing, lateral misalignment, and angular misalignment between the transmitter and the receiver coils. Data telemetry between the coils has also been measured, and all the measurement results are discussed below.
3.1. Link Efficiency. Figure 7 shows the different efficiency plots for various operating frequencies. From this figure, it is evident that the link efficiency, which is defined as the ratio of the power delivered to the receiving coil to the power supplied by the primary coil, is much higher than the overall system efficiency. At the operating frequency of $206 \mathrm{kHz}$, the overall system efficiency was found to be $12.5 \%$.

The losses in the system are contributed by the primary and the secondary unit voltage regulators and the load conditions. Table 2 tabulates the conversion and the link efficiencies of the inductive link at different operating frequencies. Figure 8 provides the frequency response curve for the link where the maximum gain has been achieved at the operating frequency of $206 \mathrm{kHz}$.

If the inductive link-based system is used in an implantable biomedical application, misalignments of the coil could occur any time resulting in the reduction in the coupling efficiency of the system. There misalignments typically can be due to change in coil spacing, lateral misalignment, or angular misalignment. In an implantable system either a single misalignment or multiple misalignments can be present. The following section describes the effect of these misalignments on the proposed inductive link. While carrying out the experiments, only one misalignment parameter is varied, while the other two parameters are kept constant to isolate the effect of each misalignment.

3.2. Coil Spacing $(d)$. The transmitter and the receiver coil are attached to separate plexiglass boards in such a way that their planes are in parallel to each other. The centers of both the coils are located along the same axis as shown in Figure 9(a). The coil spacing $d$ can be varied by keeping the transmitter coil fixed while moving the receiver coil along the axis. The mutual inductance varies inversely proportional to $d$. For this experiment, $d$ has been varied from 1 to $4 \mathrm{~cm}$. Figure 9(b) shows that as $d$ is increased beyond the rated distance of $1 \mathrm{~cm}$, the output voltage is decreased.

3.3. Effect of Lateral Misalignment $(\Delta)$. In this scenario, the centers of the coils are displaced in the horizontal direction (Figure 10(a)), which is termed as lateral misalignment $(\Delta)$. The planes of the coils are still parallel to each other. The coil spacing $d$ is kept fixed at $1 \mathrm{~cm}$, while $\Delta$ is varied from 0 to $4 \mathrm{~cm}$. Measurement results in Figure 10(b) show that increment in $\Delta$ results in decrement in output voltage. This decrement in output voltage is due to the reduction in mutual inductance which is inversely proportional to $\Delta$ [7].

3.4. Effect of Angular Misalignment $(\varphi)$. In this case, the centers of the two coils are kept along the same axis $(\Delta=0)$, but their planes are tilted to form an angle $\varphi$ (Figure 11(a)), which is termed as angular displacement. The coil spacing $d$ is kept fixed at $1 \mathrm{~cm}$, while $\varphi$ is varied from $0^{\circ}$ to $90^{\circ}$. It is observed as $\varphi$ is increased, output voltage drops due to the reduction in mutual inductance, which is inversely proportional to $\varphi$ [7]. When the planes of the coils become orthogonal to each other $\left(\varphi=90^{\circ}\right)$, there is no mutual 


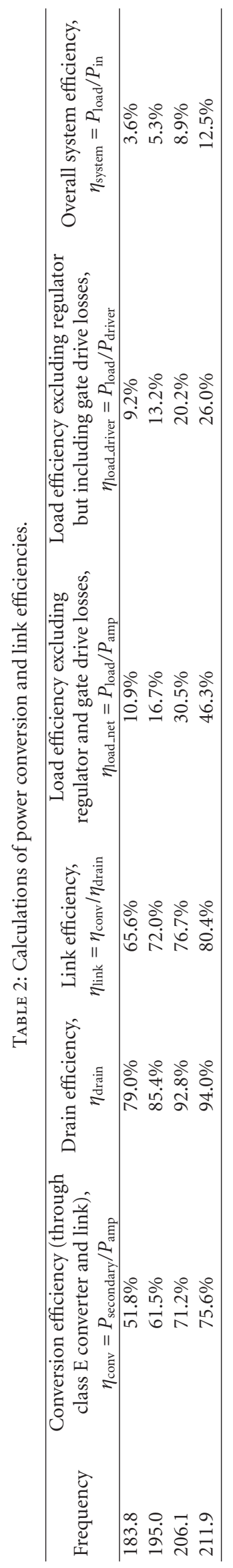




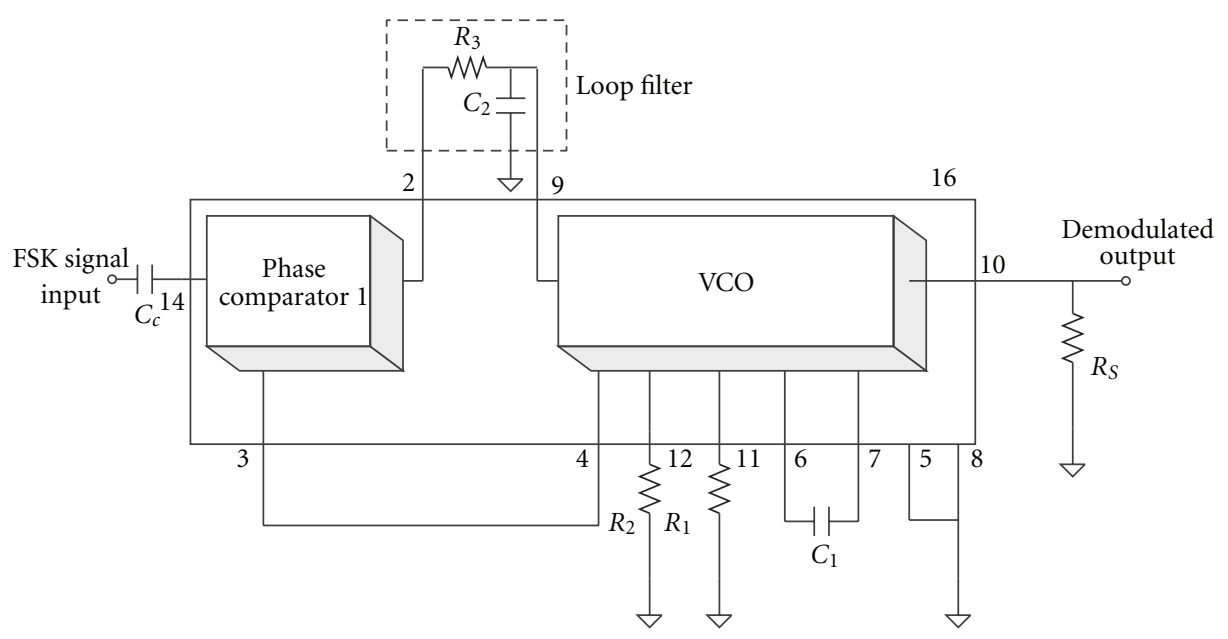

FIgURE 6: Schematic diagram of the demodulator.

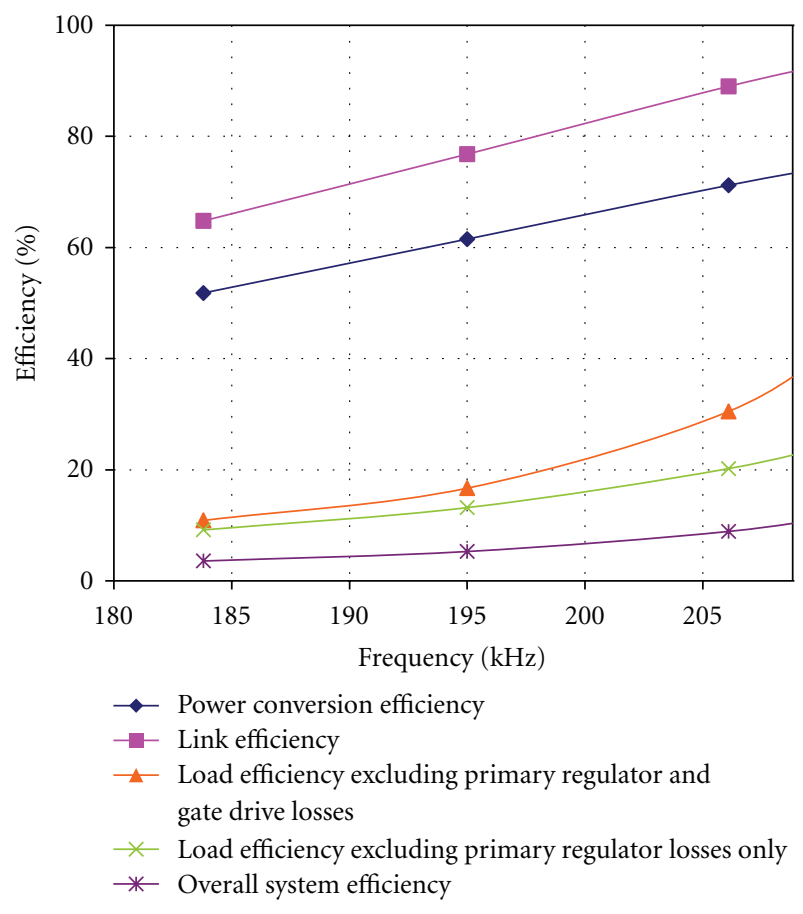

FIGURE 7: Efficiency versus the operating frequency plots.

inductance at all between the coils, and output voltage drops to zero. The test results are shown in Figure 11(b).

3.5. Data Telemetry. The modulator unit generates $121 \mathrm{kHz}$ (Mark) for logic " 1 " and $113 \mathrm{kHz}$ (Space) for logic " 0 " signal. The data signal of $1.5 \mathrm{~V}_{\mathrm{p}-\mathrm{p}}$ is generated by a sensor circuit. The data signal is then coupled with the inductive link system. To drive the $5 \mathrm{~V}$ PLL IC, the data signal is modified using operational amplifier-based amplifier. Figure 12 shows the amplified data signal and the FSK output from the

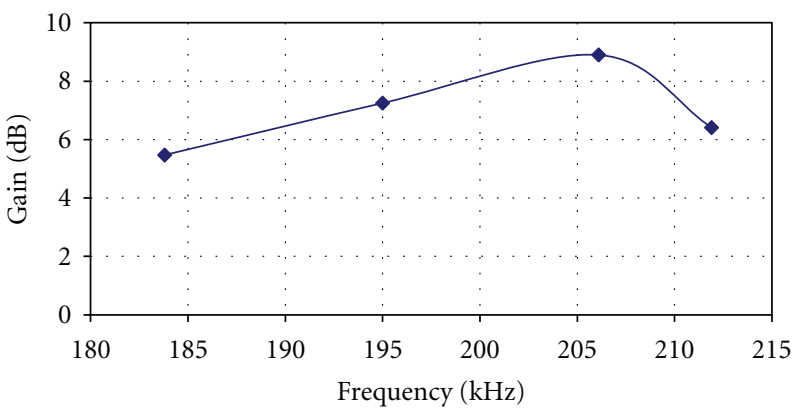

FIGURE 8: Link gain versus the operating frequency plots.

data communication unit. The frequency of the pulse signal conveys the information of the sensor current level.

For most biomedical applications, a low data rate is sufficient to meet the system requirements [35]. As indicated by link measurements, the designed backward data communication system functioned reliably up to a data rate of $10 \mathrm{kbps}$. Figure 13 shows the measured output of the demodulator for $10 \mathrm{kbps}$ data signal.

\section{Conclusion}

A wireless power transfer and backward data communication system using an inductive link has been presented. The board-level design of an inductive link was implemented using COTS components. Measurement results demonstrate power transmission of $125 \mathrm{~mW}$ with a $12.5 \%$ power link transmission efficiency. This power is sufficient to drive many low-power electronics circuit. Measurement results were demonstrated in terms of efficiency and gain at various frequencies. Test results also show the effect of the variation of coil separation, lateral misalignment, and angular misalignment. Simultaneous backward data communications were also demonstrated having a data rate of $10 \mathrm{kbps}$. Future 


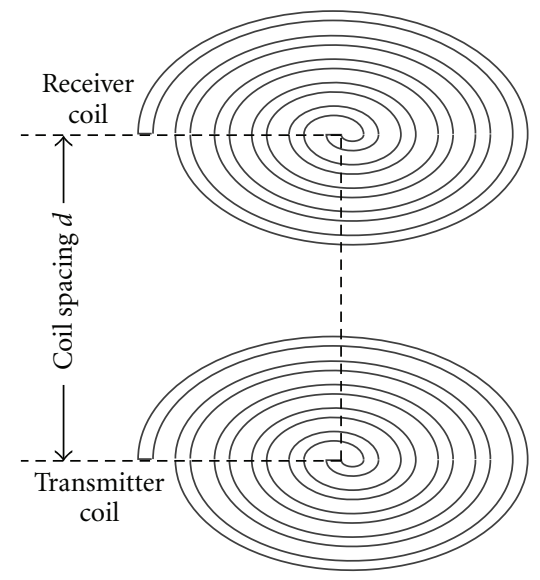

(a)

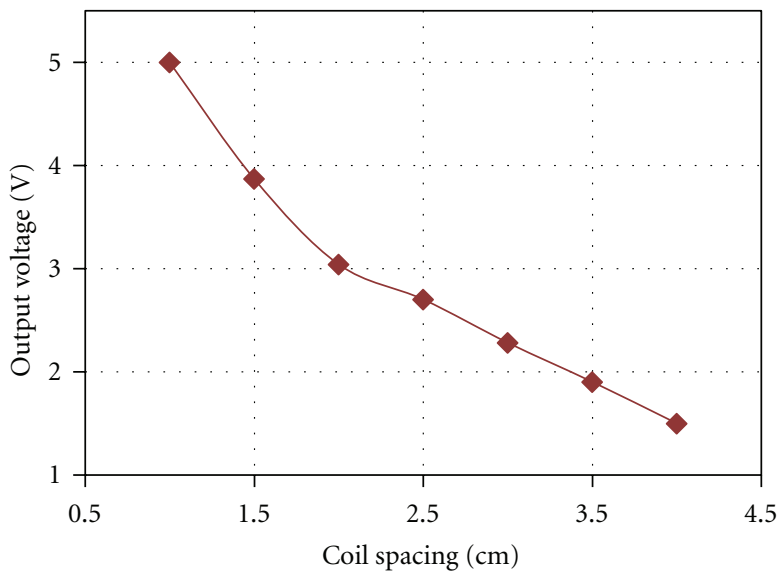

(b)

FIGURE 9: Effect of variation of coil spacing between transmitter and receiver coils: (a) orientation of the coils (b) variation of the output voltage with increase in coil spacing.

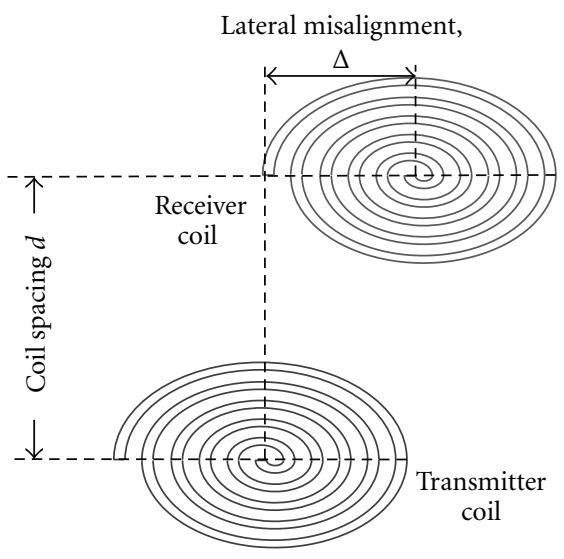

(a)

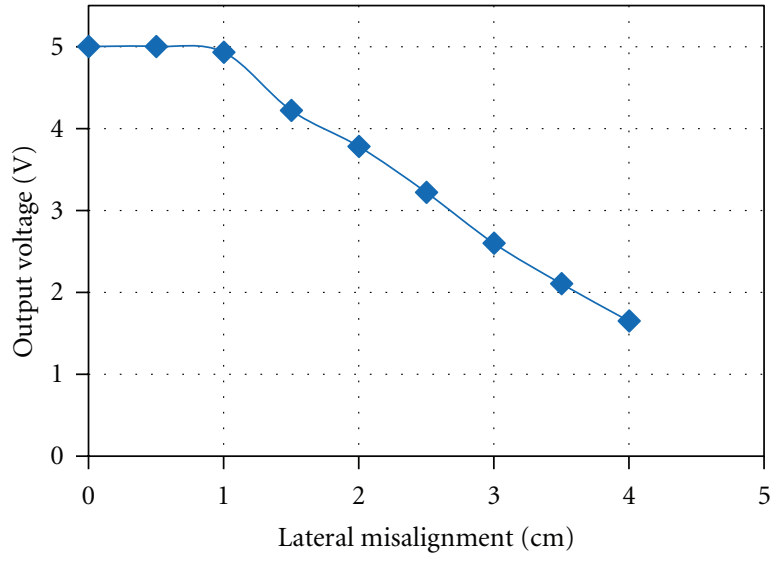

(b)

FIGURE 10: Effect of the variation of lateral misalignment $(\Delta)$ between the transmitter and the receiver coils: (a) the orientation of the coils. (b) the variation of the output voltage with increase in lateral misalignment. The coil spacing is kept fixed at $1 \mathrm{~cm}$.

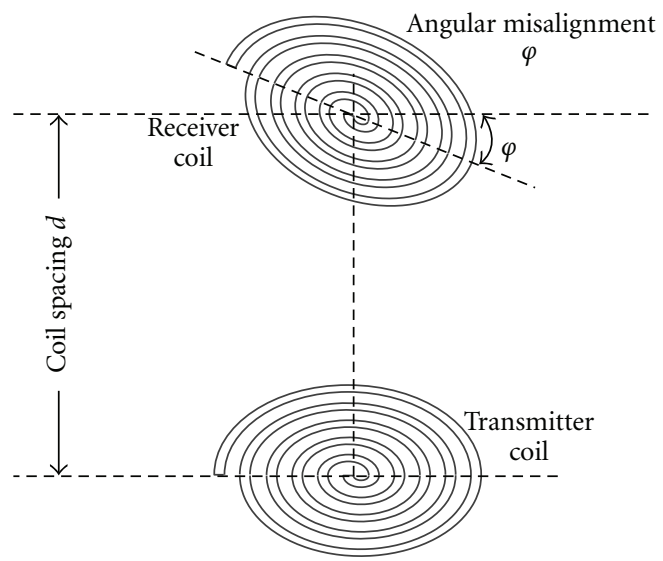

(a)

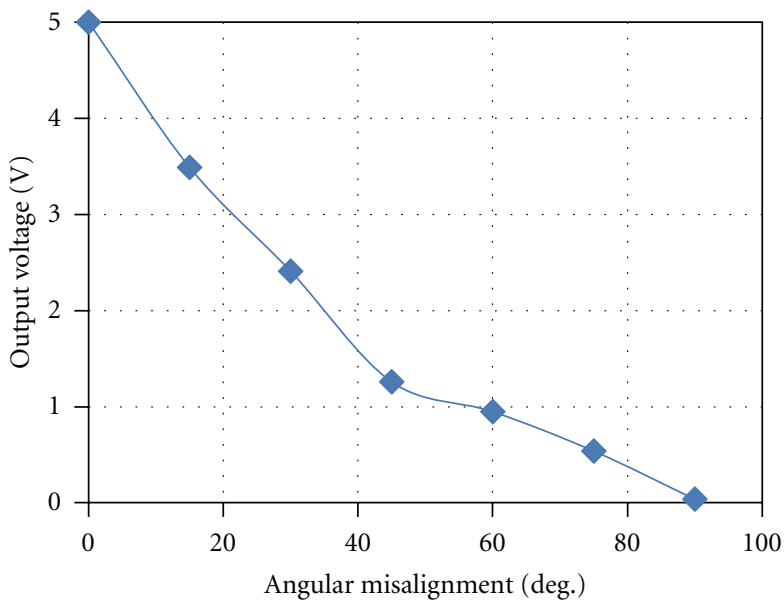

(b)

FIGURE 11: Illustration of angular misalignment $(\varphi)$ between the transmitter and the receiver coils. (a) orientation of the coils. (b) the change of output for increase in angular misalignment. The coil spacing is kept fixed at $1 \mathrm{~cm}$, and lateral misalignment is zero. 


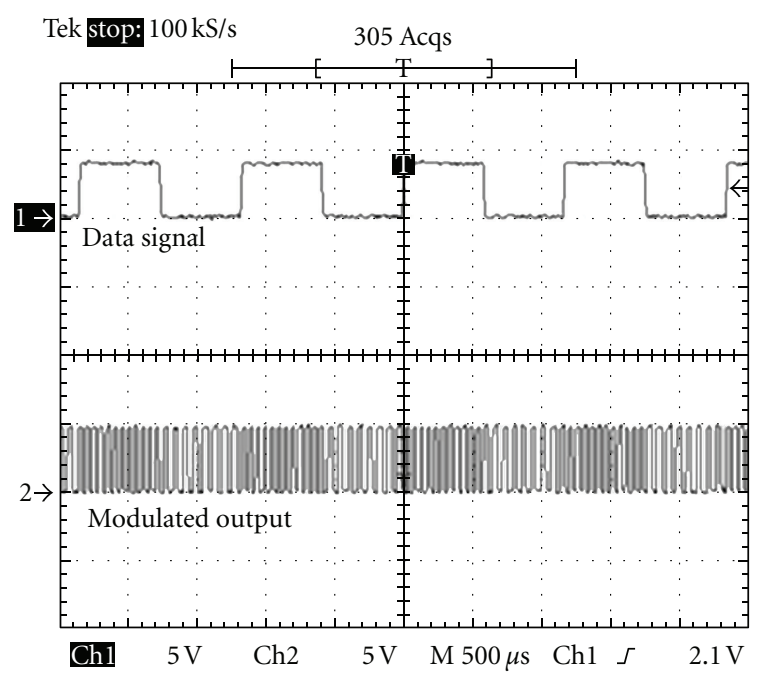

Figure 12: Amplified data signal and FSK output from the modulator unit.

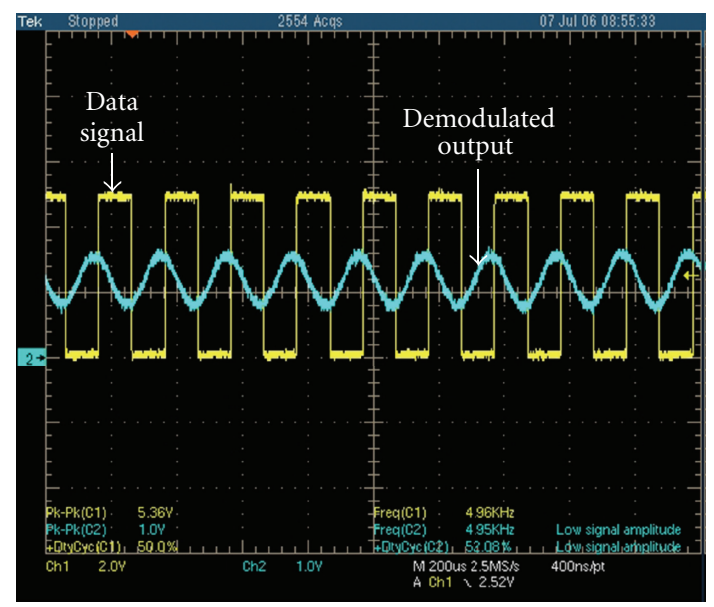

Figure 13: Demodulator output (blue) with $10 \mathrm{kbps}$ data signal (yellow).

efforts to improve the suitability of this system for biomedical implant applications would involve further miniaturization of the inductive link and improvement in the backward data communication schemes.

\section{References}

[1] R. Carta, J. Thoné, G. Gosset, and G. Cogels, "A self-tuning inductive powering system for biomedical implants," in Proceedings of the Eurosensors XXV, Athenes, Greece, September 2011.

[2] R. Bashirullah, "Wireless implants," IEEE Microwave Magazine, vol. 11, no. 7, pp. S14-S23, 2010.

[3] S. A. Jaffari and A. P. F. Turner, "Recent advances in amperometric glucose biosensors for in vivo monitoring," Physiological Measurement, vol. 16, no. 1, pp. 1-15, 1995.

[4] E. Renard, "Implantable glucose sensors for diabetes monitoring," Minimally Invasive Therapy and Allied Technologies, vol. 13, no. 2, pp. 78-86, 2004.
[5] M. Zhang, M. R. Haider, M. A. Huque, M. A. Adeeb, S. Rahman, and S. K. Islam, "A low power sensor signal processing circuit for implantable biosensor applications," Smart Materials and Structures, vol. 16, no. 2, article 034, pp. 525530, 2007.

[6] D. A. Baker and D. A. Gough, "A continuous, implantable lactate sensor," Analytical Chemistry, vol. 67, no. 9, pp. 15361540, 1995.

[7] C. Hierold, B. Clasbrummel, D. Behrend et al., "Low power integrated pressure sensor system for medical applications," Sensors and Actuators A, vol. 73, no. 1-2, pp. 58-67, 1999.

[8] G. L. Coté, R. M. Lec, and M. V. Pishko, "Emerging biomedical sensing technologies and their applications," IEEE Sensors Journal, vol. 3, no. 3, pp. 251-266, 2003.

[9] D. C. Galbraith, M. Soma, and R. L. White, "Radio-frequency coils in implantable devices: misalignment analysis and design procedure," IEEE Transactions on Biomedical Engineering, vol. 34, no. 4, pp. 276-282, 1987.

[10] W. H. Ko, S. P. Liang, and C. D. F. Fung, "Design of radiofrequency powered coils for implant instruments," Medical and Biological Engineering and Computing, vol. 15, no. 6, pp. 634-640, 1977.

[11] W. J. Heetderks, "RF powering of millimeter- and submillimeter-sized neural prosthetic implants," IEEE Transactions on Biomedical Engineering, vol. 35, no. 5, pp. 323-327, 1988.

[12] C. M. Zierhofer, I. J. Hochmair-Desoyer, and E. S. Hochmair, "Electronic design of a cochlear implant for multichannel high-rate pulsatile stimulation strategies," IEEE Transactions on Rehabilitation Engineering, vol. 3, no. 1, pp. 112-116, 1995.

[13] J. H. Schulman, "The feasible FES system: battery powered BION stimulator," Proceedings of the IEEE, vol. 96, pp. 12261239, 2008.

[14] W. Liu, K. Vichienchom, M. Clements et al., "Neuro-stimulus chip with telemetry unit for retinal prosthetic device," IEEE Journal of Solid-State Circuits, vol. 35, no. 10, pp. 1487-1497, 2000.

[15] B. Lenaerts and R. Puers, "An inductive power link for a wireless endoscope," Biosensors and Bioelectronics, vol. 22, no. 7, pp. 1390-1395, 2007.

[16] M. Guanying, Y. Guozheng, and H. Xiu, "Power transmission for gastrointestinal microsystems using inductive coupling," Physiological Measurement, vol. 28, no. 3, pp. N9-N18, 2007.

[17] S. Mandal, S. Zhak, and R. Sarpeshkar, "Circuits for an RF cochlea," in Proceedings of the IEEE International Symposium on Circuits and Systems (ISCAS '06), pp. 2845-2848, 2006.

[18] C. Sauer, M. Stanaćević, G. Cauwenberghs, and N. Thakor, "Power harvesting and telemetry in CMOS for implanted devices," IEEE Transactions on Circuits and Systems I, vol. 52, no. 12, pp. 2605-2613, 2005.

[19] M. Ghovanloo and K. Najafi, "A wideband frequency-shift keying wireless link for inductively powered biomedical implants," IEEE Transactions on Circuits and Systems I, vol. 51, no. 12, pp. 2374-2383, 2004.

[20] D. Turgis and R. Puers, "A low power radio telemetry achieving very high data rates at biocompatible frequencies," in Proceedings of the 4th International Conference on SolidState Sensors, Actuators and Microsystems, pp. 1931-1934, June 2007.

[21] M. Catrysse, B. Hermans, and R. Puers, "An inductive power system with integrated bi-directional data-transmission," Sensors and Actuators A, vol. 115, no. 2-3, pp. 221-229, 2004.

[22] M. Ghovanloo and K. Najafi, "A wireless implantable multichannel microstimulating system-on-a-chip with modular 
architecture," IEEE Transactions on Neural Systems and Rehabilitation Engineering, vol. 15, no. 3, pp. 449-457, 2007.

[23] P. Li and R. Bashirullah, "A wireless power interface for rechargeable battery operated medical implants," IEEE Transactions on Circuits and Systems II, vol. 54, no. 10, pp. 912-916, 2007.

[24] "Guidelines for evaluating the environmental effects of radio frequency radiation," Federal Communication Commission (FCC), 1996.

[25] L. S. Y. Wong, S. Hossain, A. Ta, J. Edvinsson, D. H. Rivas, and H. Nääs, "A very low-power CMOS mixed-signal IC for implantable pacemaker applications," IEEE Journal of SolidState Circuits, vol. 39, no. 12, pp. 2446-2456, 2004.

[26] R. Sarpeshkar, C. Salthouse, J. J. Sit et al., "An ultra-low-power programmable analog bionic ear processor," IEEE Transactions on Biomedical Engineering, vol. 52, no. 4, pp. 711-727, 2005.

[27] K. D. Wise, D. J. Anderson, J. F. Hetke, D. R. Kipke, and K. Najafi, "Wireless implantable microsystems: High-density electronic interfaces to the nervous system," Proceedings of the IEEE, vol. 92, no. 1, pp. 76-97, 2004.

[28] W. Liu and M. S. Humayun, "Retinal prosthesis," in IEEE International Solid-State Circuits Conference, Digest of Technical Papers, pp. 217-219, 2004.

[29] G. Vandevoorde and R. Puers, "Wireless energy transfer for stand-alone systems: a comparison between low and high power applicability," Sensors and Actuators A, vol. 92, no. 13, pp. 305-311, 2001.

[30] N. O. Sokal and A. D. Sokal, "Class E, a new class of high efficiency tuned single-ended switching power amplifiers," IEEE Journal of Solid-State Circuits, vol. SC-10, no. 3, pp. 168176, 1975.

[31] P. R. Troyk and M. A. K. Schwan, "Closed-loop Class E transcutaneous power and data link for MicroImplants," IEEE Transactions on Biomedical Engineering, vol. 39, no. 6, pp. 589599, 1992.

[32] Council on Radiation Protection and Measurements National, "Biological Effects and Exposure Criteria for Radio Frequency Electromagnetic Fields," NCRP Report 86, ISBN 0-913392-804.

[33] R. Puers, M. Catrysse, G. Vandevoorde et al., "Telemetry system for the detection of hip prosthesis loosening by vibration analysis," Sensors and Actuators A, vol. 85, no. 1, pp. 42-47, 2000.

[34] Electronic Engineer's Reference Book, Heywood, London, UK, 3rd edition, 1967.

[35] M. A. Adeeb, A class-E inductive powering link with backward data communications for implantable sensor systems, M.S. thesis, The University of Tennessee, Knoxville, Ten, USA, 2006. 

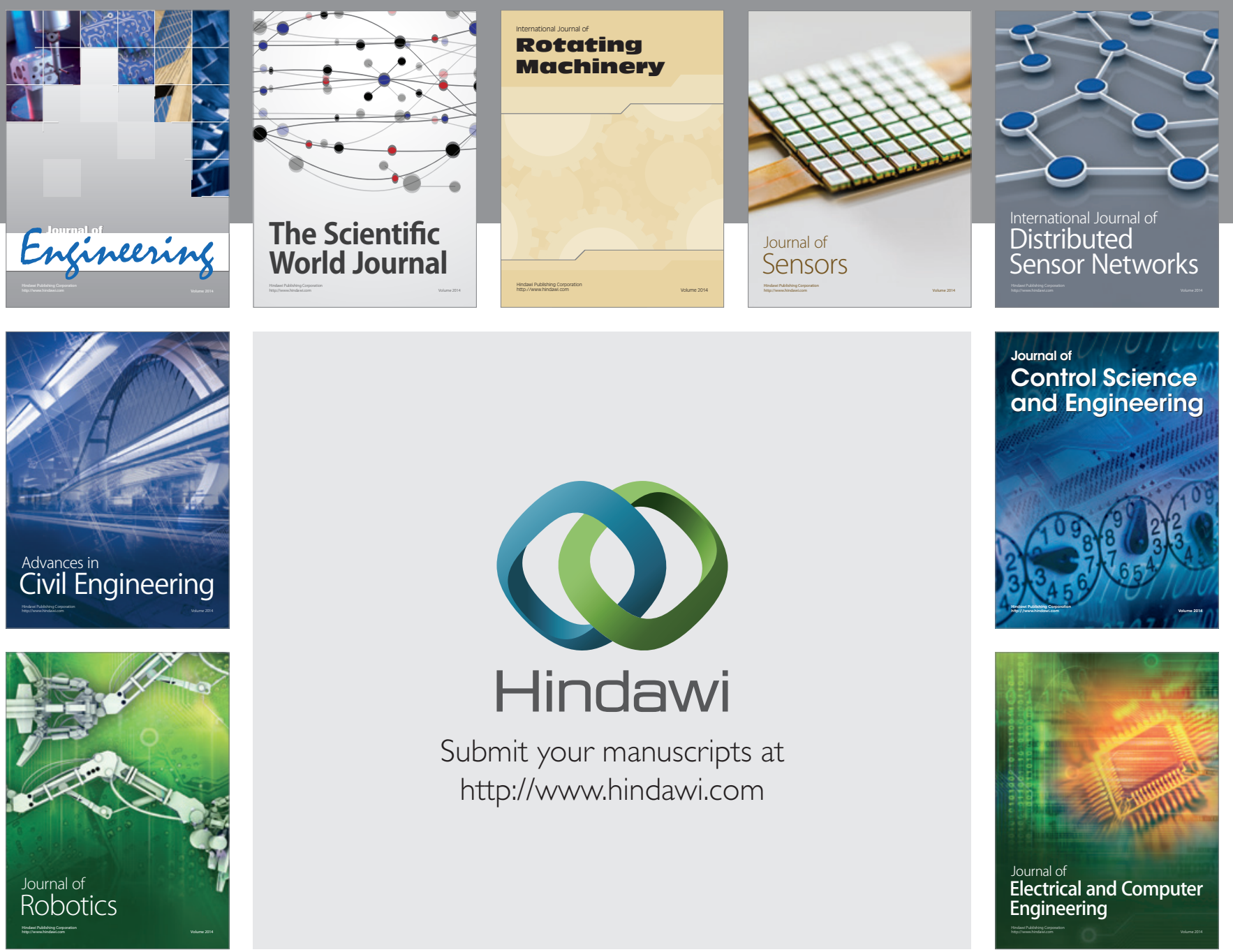

Submit your manuscripts at

http://www.hindawi.com
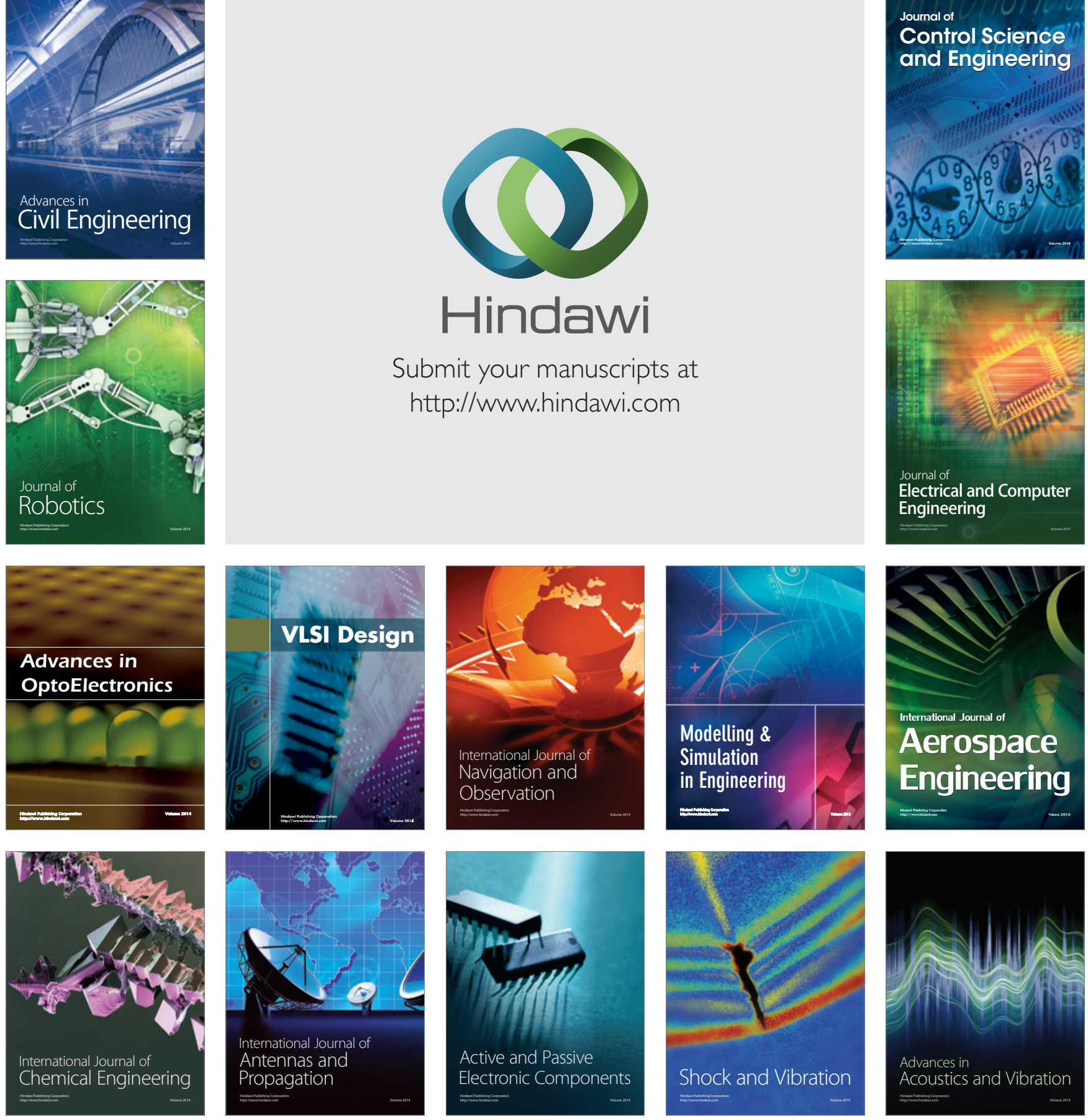